\title{
New understanding of the direct effects of spectral balance on behaviour in Myzus persicae
}

\author{
Joseph T. Fennell ${ }^{\mathrm{a}}$, Andrew Wilby ${ }^{\mathrm{b}}$, Wagdy Sobeih ${ }^{\mathrm{b}}$, Nigel D. Paul ${ }^{\mathrm{b}}$ \\ ajoseph.fennell@manchester.ac.uk \\ Jodrell Bank Centre for Astrophysics, Alan Turing Building, University of Manchester, \\ M13 9PL \\ ${ }^{b}$ Lancaster Environment Centre, Lancaster University, LA1 $4 Y W$
}

\begin{abstract}
The study of insect responses to colour has mainly focused on flying species and morphs, however colour cues are likely to be important for insect positioning within the canopy. We examine the role of illumination colour in canopy positioning of apterous Myzus persicae (Sulzer) using both a field experiment, utilising various UV-manipulating optical filters, and a laboratory experiment using video tracking of individuals illuminated by a variable intensity UVA-Blue-Green LED-array. In the field experiment, approximately twice as many aphids were located on exposed leaf surfaces under UV-deficient environments compared to UV-rich environments. The lab experiment showed all three $M$. persicae photoreceptors were involved in a visually-mediated feeding/avoidance behaviour. Highly UV-rich, greendeficient environments were up to 3 times as likely to trigger an avoidance behaviour compared to UV-absent, green-rich environments such as those found below the leaf surface. We show that apterous $M$. persicae use this, in addition to other cues, in order to locate feeding positions that minimise exposure to direct sunlight. This has relevance to both the fundamental understanding of photoprotective behaviour in Hemiptera as well as to applied research of crop production environments that disrupt pest behaviour.
\end{abstract}

Keywords: ultraviolet, UV, aphid, photobiology, Myzus persicae, behaviour, vision 


\section{Introduction}

Previous understanding of aphid responses to ultraviolet (UV) light fall broadly under either elicitation of plant defence responses (Ballaré, 2014) or the interaction with insect flight behaviour (Döring et al., 2007). Other mechanisms by which UV may affect insect survival and reproduction have been less well studied and offer both the opportunity to understand fundamental photoecology as well as opportunities for improving insect pest control in protected agriculture.

With examples from across the arthropod phylum, visual mechanisms have been shown to have a central role in navigation (Egelhaaf and Kern, 2002), host plant selection (Döring et al., 2007), predation and parasitism (Langley et al., 2006) and mate selection (Osorio and Vorobyev, 2008). Broadly, we may consider visual mechanisms to fall under two major categories: achromatic and chromatic. Achromatic vision is primarily associated with locomotion or response to moving objects, such as predators (Giurfa and Menzel, 1997). Chromatic vision is the ability to discriminate between different wavelength light and therefore requires that the insect has sensitivity to at least two different wavebands through physiologically different photoreceptors. Wavelength specificity may be achieved either through filtering the light that passes down the insect ommatidia, with wavelength-specific distal cells before it reaches the photoreceptor, or, through altering the sensitivity of the chromophore pigment in the photoreceptor cells (Briscoe and Chittka, 2001). As such, there are a very wide range of spectral sensitivities to occur across insect taxa. Whilst many Lepidoptera are tetrachromates (four photoreceptor sensitivities), the majority of Hemiptera, Diptera and Hymenoptera, like vertebrates, have trichromatic vision (three photoreceptor sensitivities). The peak sensitivities of the three bands vary somewhat, however most have a peak in the ultraviolet-A (UVA) (peak wavelength of $350 \mathrm{~nm}$ ), blue (peak wavelength of $440 \mathrm{~nm}$ ) and green (peak wavelength of 530nm) (Briscoe and Chittka, 2001).

In herbivorous insects, chromatic vision is used extensively for host finding (Doring et al., 2004; Doring and Kirchner, 2007; Fennell et al., 2019) and in flight behaviour (Barta and Horváth, 2004; Antignus, 2000). Aphids have been shown to be strongly attracted to yellow and green targets, but to be repelled by materials with high UV and blue reflectivity (Doring et al., 2004). This preference for yellow and green is likely a mechanism for detect-

ing vegetation and supports the hypothesis that aphids use a colour opponent 
strategy for host selection that is positively stimulated by green light and negatively stimulated by blue and UV light. Study of insect flight behaviour has determined, both mechanistically (Kirchner et al., 2005) and experimentally (Raviv et al., 2004), that UVA (315 nm-400 nm) is both detected and utilised for flight orientation (Pfeiffer and Homberg, 2007). During flight, insects probably use UVA to identify the sky (Barta and Horváth, 2004) due to the high degree of contrast that occurs between land and most sky conditions (Möller, 2002). Consequently, many studies have examined the impact of UV-attenuation on the spread of flying insects, due to the potential for agricultural pest control. When UV was attenuated, fewer aphids were found in polytunnel crops (Antignus, 2000; Legarrea et al., 2012b) and the population spread more slowly (Legarrea et al., 2012a), as might be expected if dispersal flight behaviour was disrupted.

From the early 1980s, there has been interest in the use of horticultural polytunnel claddings that modify the solar spectrum for pest control (Antignus, 2000). Exclusion of UV radiation through the use of UVattenuating nets had an inhibitory effect on pest Population Growth Rate (PGR): aphids and whiteflies (Order: Hemiptera) were more likely to land when they entered a UV-attenuated environment (Legarrea et al., 2012b) and, if presented with a choice, were less likely to enter areas with lower UV irradiances (Costa et al. 1999$)$ resulting in fewer infected plants and smaller pest populations in the crop as a whole. Similarly, under UV-attenuating films, thrips (Order: Thysanoptera) remained closer to their point of release and showed reduced preference for UV-attenuated environments (Kigathi and Poehling, 2012).

Whilst much work has focused on the effects of UV manipulation on migration of flying aphids into protected crop environments, little is known about how this affects wingless (apterous) morphs once a colony has established on a plant. A field experiment, using wavelength-selective filters, showed increased numbers of the aphid Aphis glycines on exposed plant surfaces under UV-opaque polythenes (Burdick et al., 2015). However it was not known if this was the result of changes in behaviour in response to different illumination, or if there was an alternative explanation (e.g. changes in plant chemistry). In order to better understand the mechanisms by which aphids select feeding sites and to test this in a different aphid species, we compared the effects of light environment on the feeding behaviours of apterous Myzus persicae in both a controlled field experiment under sunlight and in a shortterm laboratory behaviour experiment under controllable LED lighting. 


\section{Materials and Methods}

\subsection{Aphid Colonies}

Locally-collected Myzus persicae were held in culture at Lancaster University since 2010 in a climate-controlled glasshouse with an average temperature of $22.4 \pm 5.0^{\circ} \mathrm{C}$ and relative humidity of $43.5 \pm 13.4 \%$. Day:Night was $16: 8$ hours, ensuring the colony was maintained in summer state. Insects were contained in mesh tent cages $(0.5 \mathrm{~m} \times 0.5 \mathrm{~m} \times 0.5 \mathrm{~m})$ on three to five stock plants (Brassica oleracea, variety same as in experiment) per cage.

\subsection{Field Experiments}

The experiment was located on a south-facing site at Lancaster University $\left(54.05^{\circ} \mathrm{N}, 2.80^{\circ} \mathrm{W}\right)$. Nine purpose-built polytunnel structures $(3 \mathrm{~m} \times 1.3 \mathrm{~m}$ $\times 2 \mathrm{~m}$ ) were spaced $1.5 \mathrm{~m}$ apart. Each tunnel was clad in one of three commercially-available polythene claddings: Lumitherm (a Standard film with no specific UV-manipulating properties), Lumisol (a UV-transparent film) or Lumivar (a UV-blocking film). All films were produced and supplied by BPI Visqueen Ltd. Lundholm Road, Ardeer, Stevenston KA20 3NQ.

Two cultivars of Brassica oleracea L. (c.v. 'Derby Day', supplied by Marshalls Seed Ltd., Cambridgeshire, UK) and a calabrese (c.v. 'Volta', supplied by Marshalls Seed Ltd., Cambridgeshire, UK). Seeds were sown in trays of Levington's M3 compost (supplied by LBS Horticulture Ltd., Standroyd Mill, BB8 7BW) in a temperature controlled glasshouse and left to germinate uncovered. After six days, 27 plants per cultivar were transplanted into $500 \mathrm{~mL}$ pots and caged individually before 3 per cultivar were transfered to each of the nine tunnels (54 plants in total) (August). Plants were grown in the mesh cages from six days post-germination. At 23 days post-germination, five apterous (wingless) $M$. persicae were transferred to a leaf fragment in a Petri dish and placed at the base of the plant, allowing aphids to colonise the plants. Plants were harvested two weeks after inoculation with aphids (37 days post-germination) where counts of aphids were made on exposed and non-exposed parts of the plants.

\subsection{Behavioural Assays}

Calabrese (B. oleracea, c.v.'Zen' supplied by Tozer Seeds Ltd., Cobham, Surrey, UK) was used for the behavioural assays. Plants were grown in a glasshouse at Lancaster University $\left(54.05^{\circ} \mathrm{N}, 2.80^{\circ} \mathrm{W}\right)$ with supplementary illumination from 4x 600 W Senmatic FL300 Sunlight LED units. Average 
humidity was $47 \%$ and mean air temperature was $20.2 \pm 5.0^{\circ} \mathrm{C}$. Plants were grown in Levington's M3 compost and were well watered throughout the experiment. Experiments were conducted with plants 4-6 weeks after germination. Due to variation in solar radiation intensity and temperature in the glasshouse, there was some variation in size of similarly-aged plants and this was standardised by choosing similarly-sized leaves for the experimental work (those with an approximate leaf area of $25 \mathrm{~cm}^{2}$ ). Plants were isolated from exposure to aphids or other invertebrates by growing within a mesh cage after germination.

A bespoke imaging chamber (see Appendix D for full protocol) was used for all experimental work. Twelve foam squares were fixed into a $200 \mathrm{~mm} \mathrm{x}$ $100 \mathrm{~mm}$ perspex tray, which was then flooded with water. Leaf discs $(11 \mathrm{~mm}$ diameter) were removed using a punch and placed on top of the foam pads (adaxial surface facing upwards). An Light-Emitting Diode (LED) array of four high power LEDs (OSRAM GmbH Headquarters Germany, MarcelBreuer-Strae 6, 80807 Munich, Germany), driven by a microcontroller circuit, was used to illuminate the aphids in the behavioural experiment. High frequency $(100 \mathrm{KHz})$ Pulse Width Modulation (PWM) was used to vary the radiance of the four LEDs independently, allowing 21 different light treatments to be generated for the experiment Appendix E, Table E.2). For each light treatment, a mature wingless aphid was placed in the centre of each leaf disc and the tray moved into the behavioural assay chamber. Each light treatment was repeated twice (12 aphids per repeat). In all experiments, each assay was allowed to run for one hour with an image captured every 30 seconds. A proxy of feeding behaviour (movement of less than $0.014 \mathrm{~mm}$ $\mathrm{s}^{-1}$ whilst on the leaf disc, see Appendix D was measured over the 1 hour experimental period and used to generate a binary response variable. A previous study showed that, under optimal conditions, aphids spent more than $80 \%$ of time in probing or feeding behaviours (Zu-Qing et al., 2013). As such, a threshold of $80 \%$ of experiment duration was set, such that an aphid spending more than $80 \%$ of time stationary on the leaf was classified as in a 'feeding-like' behaviour and less than $80 \%$ of time was classified as in an 'avoidance' behaviour.

\subsection{Light Measurement}

Transmission spectra of polythene claddings were measured using an integrating sphere with a Macam 9910 series double monochromator spectroradiometer (Macam Photometrics Ltd.) connected to the upper port. Samples 
of polythene were placed over the entry port and illuminated with a mercury arc lamp source. The spectra were sampled at $1 \mathrm{~nm}$ resolution between 290 $\mathrm{nm}$ and $800 \mathrm{~nm}$ with an integration time of $200 \mathrm{~ms}$ to account for mains flicker. A reference spectrum was recorded for every transmission measurement and the mean of five reference and five measurement spectra were used.

Behavioural chamber measurements of irradiance were made using the same spectroradiometer with a cosine corrected head positioned at the height of the leaf disc and levelled directly upward. Spectra were measured at maximum LED PWM settings and interpolated to give spectra at different PWM settings.

Measurements of leaf transmission were made by taking seven leaves from the stock plants (B. oleracea, c.v.'Zen', as above) and placing over the cosine sensor on a bench with supplemental lighting from metal halide, UVA and UVB fluorescent tubes. The spectra were sampled at $1 \mathrm{~nm}$ resolution between $290 \mathrm{~nm}$ and $800 \mathrm{~nm}$ with an integration time of $200 \mathrm{~ms}$ to account for mains flicker. The mean of these spectra was used in further analysis.

The ASTM G173 global irradiance spectrum is a model solar spectrum for cloudless skies, representing the global irradiance at each wavelength, averaged across season and latitude in North America ASTM International, 2012). In this study it is used to estimate insect-visual colour coordinates of sunlight and filtered sunlight, independently of total irradiance.

\subsection{Aphid visual colourspace}

The Visual Action Spectra (VAS) for each of the $3 M$. persicae photoreceptors was taken from published data (Döring et al., 2007). These were generated by electroretinography (ERG) and are a fitted function describing the relative response of each type of photoreceptor at a given photoreceptor. Each photoreceptor VAS was max-normalised to one.

In order to test the effects of amplitude (integrated response over all photoreceptors) and colour separately (the response of a photoreceptor, proportional to the sum of photoreceptors), we define a colourspace using an orthogonal basis transform of the integrated photoreceptor responses, similar to that defined by Osorio and Vorobyev (2008). Using the photoreceptor response spectrum $R_{i}(\lambda)$ generated by ERG, we define the response of the $i$ th colour receptor type $\left(P_{i}\right)$ as:

$$
P_{i}=\int_{300}^{700} R_{i}(\lambda) S(\lambda) d \lambda
$$


where $S(\lambda)$ is an irradiance spectrum that stimulates the receptor. In the case of sunlight filtered by a polythene, we define $S(\lambda)$ using the transmission spectrum of the polythene $T(\lambda)$ and the model global sunlight spectrum ASTM G173 $(M(\lambda))$.

$$
S(\lambda)=T(\lambda) M(\lambda)
$$

In the case of the behavioural chamber, $S(\lambda)$ was the measured irradiance spectrum in the chamber.

For a trichromate, we can fully represent any visual stimulus with three components: the amplitude of the overall signal $(A)$ and any two of the possible three colour coordinates which in this case are defined as:

$$
\begin{gathered}
A=\sum_{i=1}^{n} P_{i} \\
c_{x}=\frac{P_{\text {long }}}{A} \\
c_{y}=\frac{P_{\text {short }}}{A} \\
c_{z}=\frac{P_{\text {mid }}}{A}
\end{gathered}
$$

We choose the long-wavelength ('green') $c_{x}$ and short-wavelength ('UV') $c_{y}$ coordinates to represent the chromatic information, along with the amplitude (' $A$ ') to represent the intensity of the signal. The amplitude can be considered the total aphid photoreceptor-weighted irradiance, equivalent to the plant-weighted irradiance presented previously (Paul et al. 2005). We do not include the third coordinate (in this case $c_{z}$ ) in the model fitting process as it is a linear combination of the other two. E.g. by substitution:

$$
c_{z}=1-c_{x}-c_{y}
$$

\subsection{Statistical Methods}

All statistical analyses were carried out in the Python programming language using the 'pymc3' package (Salvatier et al., 2016). Generalised Linear Models (GLMs) were constructed to model the parameter distributions for 
the responses measured during the experiment. We chose a Bayesian approach, representing the coefficients in the model as unknown distributions with very wide ('weakly-informative') priors. Sampling the parameter space allows reconstruction of these distributions and the posterior mean and credible intervals (the Bayesian equivalent of confidence intervals) to be estimated. Different response variables have different likelihood distributions which are chosen a priori. For count data of biological populations, due to overdispersion (variance greater than the mean) the negative binomial distribution with a log link function was used to model the likelihood (as discussed in Ver Hoef and Boveng, 2007). For binary responses (e.g. 'feeding-like' versus 'avoidance'), the binomial distribution with a logit link function was used to model the likelihood. For all models, the pymc3's default extension to the Hamiltonian Monte Carlo ('No U-Turn' or NUTS) algorithm was used to sample the parameter space. Unless otherwise stated, the default weaklyinformative priors were used in accordance with the published documentation (Salvatier et al., 2016).

Interpretation of the models is expressed in terms of effect sizes and their distributions, as estimated using the sampling approach described above. As we do not know the true distribution of the parameters, we present the most probable (posterior mean) estimate of a parameter and the region in which $95 \%$ of the samples lie (the $95 \%$ credible interval). In general, if the $95 \%$ credible interval of the effect size does not overlap zero, the probability that there is a non-zero effect of a treatment is greater than or equal to 0.95 , and would be considered significant under explicit 'tail tests'. It should be noted that the effect sizes are in the 'link-scale' of the respective GLM.

\section{Results}

\subsection{Light Environments}

The peaks sensitivities of the three Myzus persicae photoreceptors, recovered from Doring et al. (2004), were at $330 \mathrm{~nm}$ ('short'), $450 \mathrm{~nm}$ ('Mid') and $530 \mathrm{~nm}$ ('Long') (Figure1.A). The polythene cladding had similar PhotosyntheticallyActive Radiation (PAR) transmission (Lumivar: 80\%, Lumitherm: 81\%, Lumisol: 83\%) but had different UV transmission properties (Figure 1.B). UV-opaque film ('Lumivar') had the lowest transmission of UV (UVB: < 0.1\%, UVA: $1.6 \%$ ), Standard ('Lumitherm') had an intermediate transmission (UVB: 0.1\%, UVA: 28.3\%) and UV-transparent film ('Lumisol') had the highest (UVB: $75.6 \%$, UVA: $78.9 \%$ ). 
Measurement confirmed that the LED units had peak wavelengths at 370 $\mathrm{nm}, 448 \mathrm{~nm}, 526 \mathrm{~nm}$ and $674 \mathrm{~nm}$ (Figure 1.C) and so by dimming each LED separately, allowed a very wide range of different spectral balances. This covered all likely field scenarios achievable by filtering.

\subsection{Field experiment}

Aphid counts were made on leaf materials immediately after harvest from the tunnels (Figure 2.A and 2.B). The count data were overdispersed (variance greater than the mean) and so were modelled with a negative binomial distribution. Different polythenes and cultivars were treated as separate classes, each with an associated coefficient, and the full additive model was fitted for both total plant count and exposed feeding position count. There was no difference in total population between treatments (Figure 2.A). The range of effect sizes for all light treatments was very high and overlapped zero in all cases (see Appendix F, Table F.3) indicating no statistical difference in total, final population size.

Exposed positions were defined as leaf surfaces visible from above. Populations under UV-opaque polythenes had larger populations on exposed leaf surfaces than populations under UV-transparent polythenes (posterior predictive mean: $230 \%$ increase compared to UV-transparent, effect size: 1.19, $95 \%$ credible interval: 0.63 - 1.75, Figure 2.B). Under the Standard polythene treatment, there was a marginal increase in the number of insects found on exposed leaf surfaces compared to under UV-transparent polythenes (posterior predictive mean: $74 \%$ increase, effect size: $0.55,95 \%$ credible interval: -0.04 to 1.135, Figure 2.B). Due to the small number of observations ( $n=9$ per treatment), we do not draw strong conclusions from these data but used them to form the hypothesis for the next section.

\subsection{Aphid photoreceptor responses}

Aphid photoreceptor responses were estimated as described above for all light treatments (see Appendix E.2) and the range of experimental treatments fully covered the range of treatments used in the field experiments (Figure 1.D). As the field treatments predominantly varied in the $c_{y}$ (shortwavelength/UV) coordinate, the LED experimental treatments also covered a much wider range of possible light environments by allowing wide variation in the $c_{x}$ (long-wavelength/Green) coordinate (Figure 1.D). The longwavelength coordinate $\left(c_{x}\right)$ ranged from 0.233 to 0.782 and the short-wavelength coordinate $\left(c_{y}\right)$ ranged from .001 to .101. Amplitudes ranged from 2.75 to 

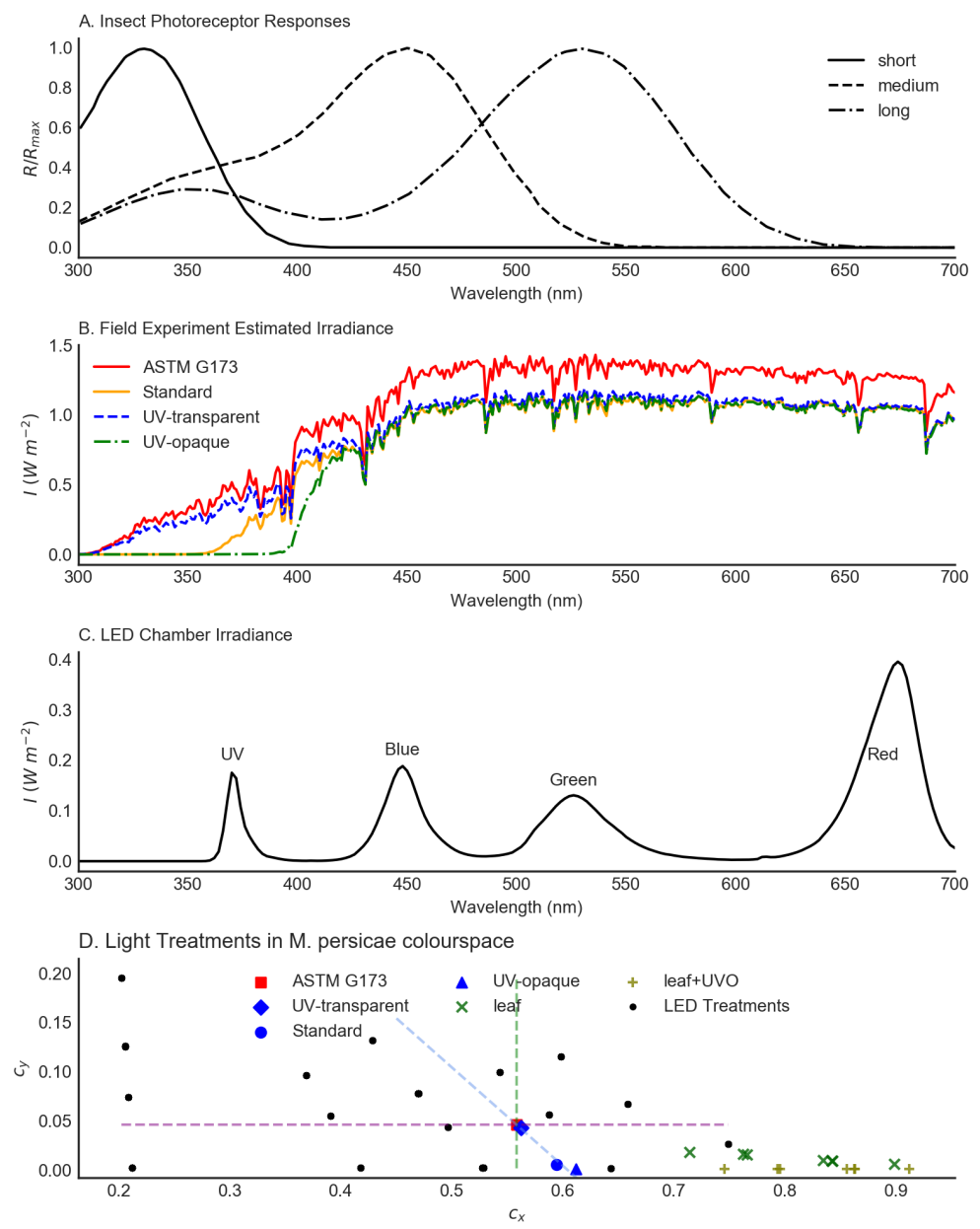

Figure 1: Spectra for (A) the short, medium and long photoreceptors of Myzus persicae (as presented in Doring et al. (2004)),(B) The ASTM G173 irradiance spectra of light under the three polythene films used in the field experiment and $(\mathrm{C})$ the leaf-level irradiance at maximum power setting for all LEDs with each peak labelled by the corresponding LED. (D) Light treatments for laboratory and field experiments as a function of $c_{x}$ and $c_{y}$ in aphid colour coordinates. The intersection of the three dashed lines shows sunlight with each line showing constant short photoreceptor -response (coloured purple), constant midphotoreceptor response (coloured blue) or constant long-photoreceptor response (coloured green). Additional positions are plotted for the model solar spectrum (ASTM G173), solar spectrum filtered by 3 polythenes (UV-transparent, Standard and UV-opaque), solar spectrum filtered by B. oleracea leaf (leaf) and solar spectrum filtered by Brassica oleracea leaf and UV-opaque polythene (leaf+UVO). 

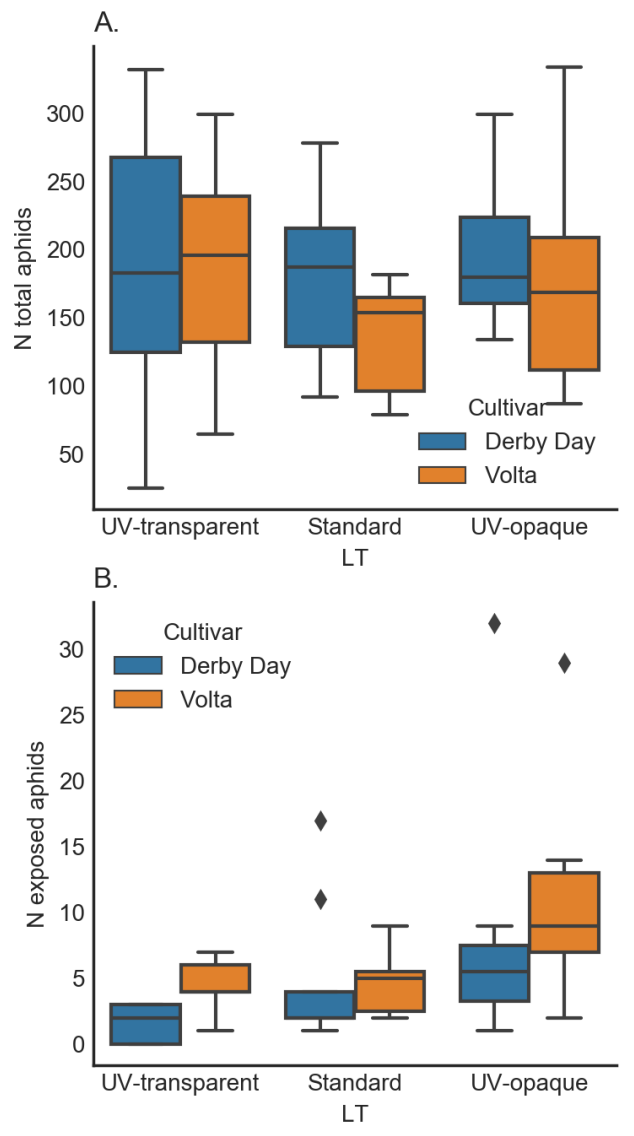

Figure 2: Boxplot of (A) total Myzus persicae per plant and (B) total in exposed positions. Central horizontal line shows the median and whiskers represent the $95 \%$ confidence interval. Outliers are shown as points. 


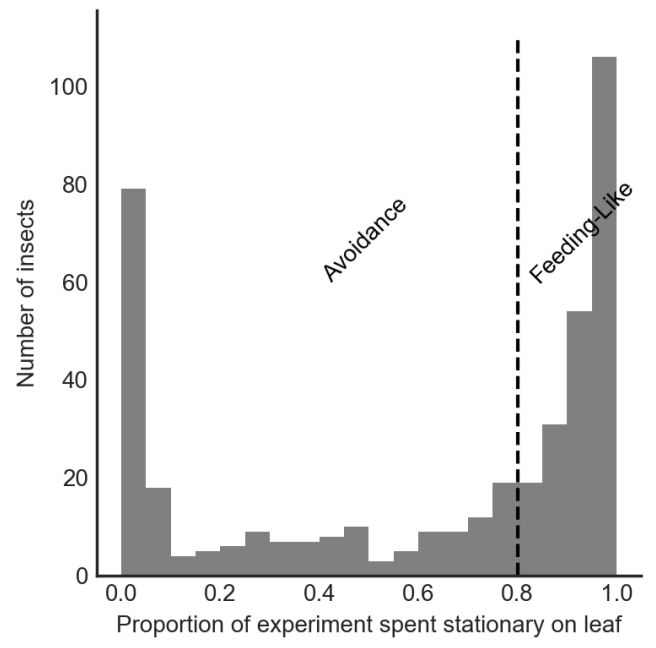

Figure 3: Histogram of proportion of experiment spent in a stationary position on leaf for each aphid (Myzus persicae). Dashed line shows threshold between avoidance $(<80 \%$ of experiment in a stationary position on leaf) and feeding-like behaviour ( $>80 \%$ of experiment in a feeding position on leaf)

12.55. As such we could reliably test responses in the long-wavelength coordinate $\left(c_{x}\right)$ and the short-wavelength coordinate $\left(c_{y}\right)$.

\subsection{Aphid behavioural response to colour}

The distribution of aphid responses to the different light treatments tended towards binary (Figure 3): aphids tended either to respond negatively to the environment, or settle and begin feeding for the duration (1 hour) of the experiment. This was as expected and supported the previous study that showed aphids spend more than $80 \%$ of time in probing or feeding behaviours (Zu-Qing et al., 2013).

Using amplitude of photoreceptor response $(A)$ and the long- and shortwavelength colour coordinates $\left(c_{x}, c_{y}\right.$, respectively), different statistical models to describe the observed behaviour were compared Appendix F, Table F.5). The likelihood was modelled with a binomial distribution and can be interpreted as the probability that an aphid is observed in an avoidance behaviour given the light treatment. Model comparison using the Widely Applicable Information Criterion (WAIC) showed that the observed data were best described by a model using the colour coordinates $c_{x}, c_{y}$ and not the amplitude $(A)$ Appendix F, Table F.5): 


$$
y=\operatorname{logit}^{-1}\left(\beta_{0}+\beta_{1} c_{y}+\beta_{2} c_{x}\right)
$$

Effect sizes are presented in log-odds units (the 'link-scale' of the binomial GLM) so for ease of interpretation, the posterior predictive distributions were sampled to provide estimated probabilities of avoidance behaviour $\left(P_{A}\right)$ (Figure 4).

Light environments that caused proportionally more stimulation of the long-wavelength photoreceptor (i.e. high $c_{x}$ values) decreased the probability of avoidance behaviour $\left(\beta_{2}=-1.30,95 \%\right.$ Credible Interval: -2.49 to -0.07 , Figure 4.A). Light environments that caused proportionally more stimulation of the short-wavelength photoreceptor (high $c_{y}$ values) was found to have a larger and opposite effect with more stimulation increasing the probability of avoidance behaviour $\left(\beta_{1}=16.36,95 \%\right.$ Credible Interval: 8.41 to 24.10 , Figure 4.B).

The highest value of $P_{A}$ was under high short-wavelength stimulation and low long-wavelength stimulation $\left(P_{A}>0.8\right.$, Figure 4.C). Under conditions when there was no stimulation of the short-wavelength photoreceptor (i.e. no UV light), low long-wavelength stimulation and therefore high midwavelength or 'blue' stimulation had higher avoidance probabilities $\left(P_{A} \approx\right.$ 0.51) compared to avoidance probability under light conditions with high long-wavelength stimulation $\left(P_{A} \approx 0.30\right.$, Figure 4.C)

\subsection{Estimation of responses under real-world light environments}

Using the measured transmission spectra for polythenes and B. oleracea leaves, and the model described above, $P_{A}$ was calculated for the ASTM G173 sunlight model filtered through each of these optical filters (Table 1, Figure 4.C). Aphids in full sunlight were the most likely to exhibit an avoidance response $\left(P_{A} \approx 0.53\right)$. Under polythenes, aphids under UV-transparent were predicted to have the highest probability of avoidance $\left(P_{A} \approx 0.52\right)$ with reduced probability under standard $\left(P_{A} \approx 0.36\right)$ and UV-opaque $\left(P_{A} \approx 0.34\right)$. Under $B$. oleracea leaves, the mean estimate was $P_{A} \approx 0.32$ for solar UV and so was broadly comparable to under standard and UV-opaque polythenes. Under UV-opaque polythene, the under-leaf estimate was slightly lower $\left(P_{A} \approx 0.28\right)$. 

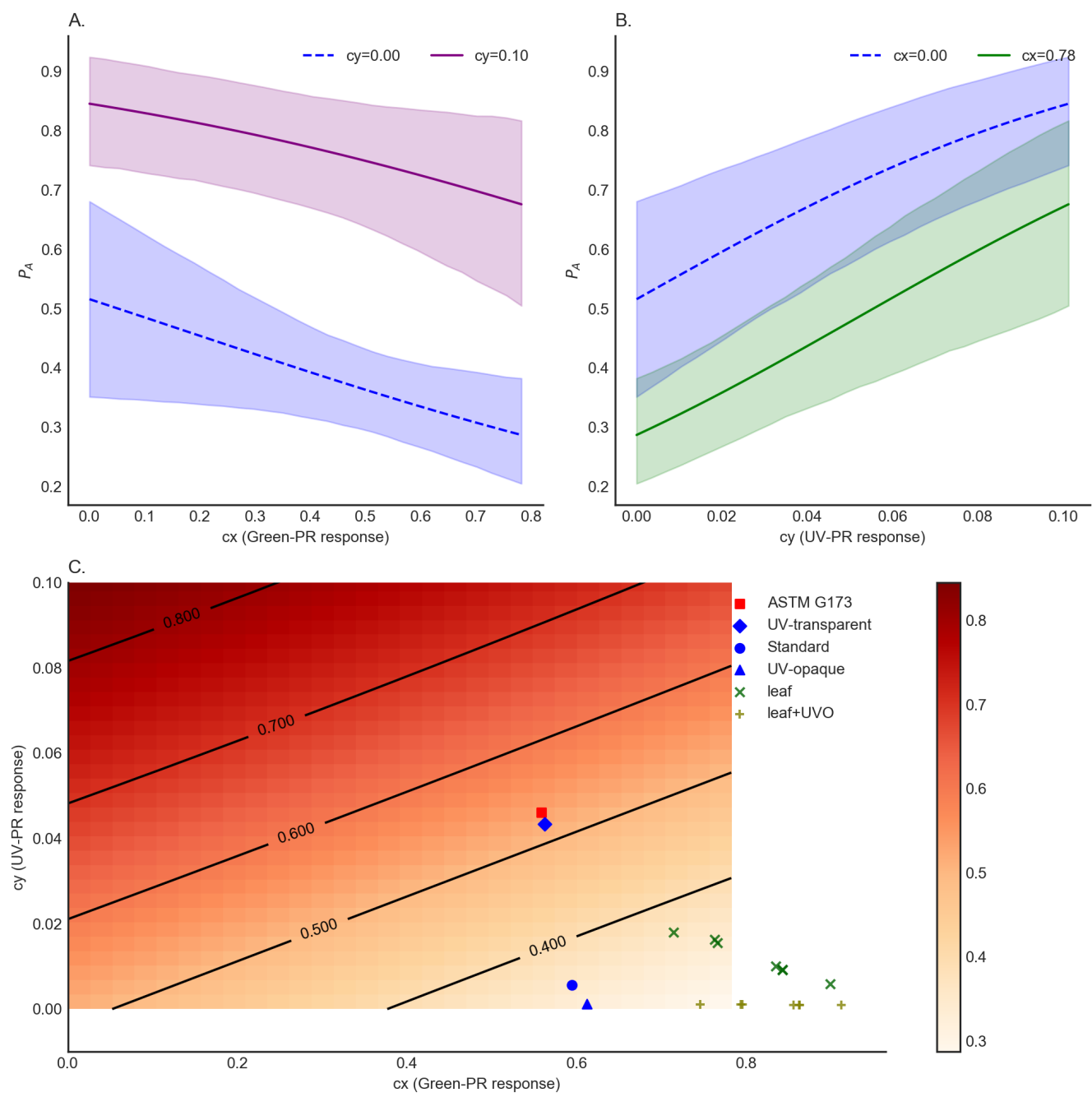

Figure 4: Posterior predictive distribution for Myzus persicae behavioural response to illumination colour. (A) Probability of avoidance behaviour $\left(P_{A}\right)$ as a function of longwavelength $\left(c_{x}\right)$ response for 2 extremes of $c_{y}$ sampled by the experiment shows the $2 \mathrm{D}$ parameter space with probability of avoidance as a function of short-wavelength $\left(c_{y}\right)$ and long-wavelength $\left(c_{x}\right)$ responses. (B) $P_{A}$ as a function of long-wavelength $\left(c_{y}\right)$ response for 2 extremes of $c_{x}$ sampled by the experiment. Shaded regions in (A) and (B) show the $95 \%$ credible intervals. (C) shows $P_{A}$ as a $2 \mathrm{D}$ function of short-wavelength (cy) and long-wavelength (cx) responses. Additional point estimates are plotted for the model solar spectrum (ASTM G173), solar spectrum filtered by 3 polythenes (UV-transparent, Standard and UV-opaque), solar spectrum filtered by Brassica oleracea leaf (leaf) and solar spectrum filtered by Brassica oleracea leaf and UV-opaque polythene (leaf+UVO). 
Table 1: Estimated probability of avoidance behaviour $\left(P_{A}\right)$ for unfiltered ASTM G173 solar spectrum; ASTM G173 filtered through different polythenes ('UV-transparent' Lumisol, 'Standard' - Lumitherm, 'UV-opaque' - Lumivar); ASTM G173 filtered through Brassica oleracea leaves; and ASTM G173 filtered through B. oleracea leaved and UVopaque polythene

\begin{tabular}{lr}
\hline Light Environment & $P_{A}$ \\
\hline ASTM G173 & 0.53 \\
UV-transparent & 0.52 \\
Standard & 0.36 \\
UV-opaque & 0.34 \\
Leaf (mean) & 0.32 \\
Leaf + UV-opaque (mean) & 0.28 \\
\hline
\end{tabular}

\section{Discussion}

The results presented here provide novel evidence that $M$. persicae uses three photoreceptors, not only for flight behaviours in winged morphs (Chyzik et al., 2003; Döring et al., 2007), but also as an important component of the environmental perception mechanism of wingless (apterous) morphs. The best model describing the relationship between light and behaviour demonstrated that all three $M$. persicae photoreceptors are involved in the lightmediated feeding/avoidance response and act in opposition to each other. Long wavelengths promoted feeding, whilst short wavelengths promoted avoidance behaviours. The light environments with the lowest probability of avoidance coincided with the predicted light environment in shaded parts of the $B$. oleracea canopy (Figure 4.C) and so we propose that direct perception of illumination colour is used by apterous aphids to locate shaded feeding positions, for which they have a preference (Figure 2).

\subsection{Interpretation of statistical models for visually-mediated feeding behaviour}

Our results show that $M$. persicae apterae are more sensitive to changes in ultraviolet light than longer wavelengths (Figure 4) and respond with an avoidance behaviour as the colourspace becomes biased towards short wavelengths. The best fitting model was independent of amplitude and indicated that all three aphid photoreceptors were involved in determining the behaviour. This is consistent with previous studies at both an experimental 
(Chittka et al., 1992) and mechanistic (Borst, 2009) level that have shown most insects use a colour opponent mechanism: a negative feedback system that allows perception of colour, independent of amplitude. This allows organisms to perceive chromatic signals over widely varying irradiances as would be experienced at different times of the day.

We performed model comparison across a range of candidate models (Table F.5). The most probable model is presented to describe $M$. persicae feeding behaviour in response to changes in spectral balance, however an alternative model that also included the amplitude (total aphid-weighted irradiance) of the stimulus was only slightly worse-performing (see Table F.5). When amplitude was included, it had a very small effect on predicted responses over the relatively small range of amplitudes possible in our experiment ( 0.1 to $12.55 \mathrm{~W} \mathrm{~m}^{-2}$ in laboratory compared to a maximum of $\sim 280 \mathrm{~W} \mathrm{~m}^{-2}$ in the field). Therefore, whilst we can present a strong case that colour balance is the most important mechanism, we cannot rule out an interaction with amplitude at higher intensities than were tested in this experimental work.

\subsection{UV-Green opponency for avoidance of $U V$}

In the controlled behaviour experiment, apterous (wingless) female $M$. persicae spent less time in feeding-like behaviour under UV-rich light environments than under UV-deficient environments (Figure 4.B). It was also observed that aphids under high UV treatments sometimes circled the edge of the leaf disc (see videos in Appendix C). We interpret this as the same avoidance that was observed in the field but constrained, because the assay prevented movement to the underside of the leaf. The pattern of behaviour supported the findings in the field study where more aphids were located in exposed parts of the plant under low UVA treatments. As such, we find strong evidence that preference for shaded feeding sites is determined by perception of solar radiation. Based on our model of visually-mediated feeding/avoidance behaviour, it was hypothesised that this positioning was as a direct response to UV perception by the aphid, causing them to move from exposed (typically the upper surfaces of leaves located higher in the canopy) to more shaded parts of the canopy. This preference for shaded leaf surfaces has also been demonstrated in at least one other aphid species (Aphis glycines Burdick et al. (2015)) and also in the spidermite Panonychus citri, which showed reduced oviposition preference for upper leaf surfaces exposed to full sunlight (Fukaya et al., 2013). 
Avoidance of high UV environments is likely to be advantageous to apterous aphids. Feeding sites high in aphid-visible UV are also likely to be exposed to higher levels of shorter wavelength UV (ultraviolet-B (UVB)). Field-like UV doses caused increases in mortality in Hemiptera (Burdick et al. 2015, Tariq et al. 2015), however these studies did not isolate the direct effect on the insect from potential indirect effects mediated through the plant (Ballaré, 2014). Short-term exposure of apterae to environmentally relevant UVB doses on a non-plant substrate increased mortality in $M$. persicae (Fennell, 2016) demonstrating that there is likely to be strong positive selection for short wavelength avoidance behaviours. However whilst M. persicae were negatively affected by exposure to UV, other species may be more tolerant to living on exposed leaf surfaces. This tolerance may be more likely when other, competing selection pressures outweigh the harmful effects of UV exposure, driving physiological adaptation. Movement to the upper surface of the leaf was shown to be advantageous for the aphid Melanocallis caryaefoliae when predation risk was high as it reduced contact with predators (Paulsen et al., 2013).

Other invertebrates, such as spidermites, also balance UV exposure with pther biotic and abiotic stresses (Sakai et al., 2012; Fukaya et al., 2013; Ohtsuka and Osakabe, 2009; Onzo et al., 2010). The majority of these studies used UVB doses comparable to field UVB day doses, however field-like UVA doses were also shown to affect egg survival in at least one species (Onzo et al. 2010). Therefore whilst the effects of UV on survival and fecundity are likely to be driven largely by shorter wavelength UVB, UVA may also have a direct effect.

\subsection{Green-Blue opponency for host finding}

A second opponent mechanism was also identified in apterous aphid feeding behaviour: Green(long)-blue(mid) opponency occurred in the absence of UV where aphids showed increased probability of avoidance behaviours under higher mid-wavelength (blue) photoreceptor stimulation (Figure 44). Bluebiased light environments are relatively unusual for an aphid as the foliage absorbs most blue light and is either transmissive or reflective of green light. Reducing the proportion of green light in the illumination spectrum reduces the relative proportion of green light reflected off a leaf surface, therefore making it appear less 'leaf-like' to the insect. Identification of plant material by its high long-wavelength saturation and high contrast with the background 
has been previously identified as a mechanism by which alate aphids first locate a potential host, before using other cues (tactile, exploratory probing, etc.) to establish the suitability for extended feeding (Doring et al., 2004). Apterous aphids may, therefore, also use green-blue balance to differentiate plant from non-plant, and so if the illumination causes the plant material to be substantially different to leaf material, aphids may reduce their feeding effort and increase movement.

It is also possible that blue-biased light environments cause aphids to incorrectly identify the defensive status of the plant. Anthocyanins have been shown to be visual indicators of phenolic status as they have high pleiotropy with other more toxic flavonoids (Johnson and Dowd, 2004). Leaf tissue with low anthocyanin content is highly reflective in the green and less reflective in the blue, whereas leaf tissue with higher anthocyanin content reflects proportionally more blue light (Gitelson et al., 2009). Therefore, illumination of leaves with blue-biased light may make them appear higher in anthocyanins and so act as a feeding deterrent, however more work is needed in this area to test this.

\subsection{Applying the colourspace model to predict behaviour in crop production environments}

The approach used in this paper, where $M$. persicae behavioural responses were mapped to the coordinates within its trichromatic colourspace, is a powerful tool for predicting apterous responses to different light environments. The responses of hemipteran pests to light environments under horticultural polythene films are of particular interest to this study, due to the implications for their use in pest control. As such, the simulated light environments within polytunnels clad with various spectrally-modifying polythene films were used to generate predictions of aphid behavioural response (Figure 4). Using a simple metric for aphid tolerance - the probability of a feeding response - this study showed that $M$. persicae may be as tolerant of exposure to sunlight filtered by UV-opaque films as fully-shaded feeding sites within a plant under full sunlight. An aphid on an exposed site under these polythenes would be expected to perceive the light environment as though it were a shaded site and more readily accept it as a feeding site.

The field experiment confirmed that more $M$. persicae fed on the exposed upper leaf surfaces of Brassica oleracea under the UV-opaque polythene than under the UV-transparent polythene (Figure 2) and this was supported by 
the relative predicted preference of the different polythenes (Table 11). Additionally, the laboratory experiment indicated that the same probability of avoidance should be expected under UV-opaque polythenes as in shaded parts of a canopy in unfiltered sunlight (Figure 4, Table 1), however the percentage of aphids feeding on the exposed leaf surface under UV-opaque polythene was much lower than on shaded locations in the canopy under UV-transparent polythene. Although the model predicts a slightly higher probability of avoidance on exposed, compared to shaded, feeding sites under UV-opaque polythene (0.32 c.f. 0.28), the large differences between exposed and shaded populations observed in the field are likely due to additional, non-visual mechanisms that operate alongside the mechanism proposed in this study. Whilst short term decisions about attempted feeding are controlled by the illumination colour, insects may withdraw the stylet and probe more frequently when vessels are not located (Hardie et al., 1992), or when the vessels or plant tissue contain elevated concentrations of plant defensive compounds (Golawska et al., 2012; Rangasamy et al., 2015; Zu-Qing et al., 2013). Hemiptera may also respond to tactile cues on the leaf surface, which may influence feeding frequency or duration Simmons (1999). If these properties vary across the plant, these mechanisms would also be expected to influence the distribution of feeding aphids, alongside the visually-mediated avoidance/feeding mechanism proposed in this study.

\section{Conclusions}

We have demonstrated $M$. persicae uses colour information for positioning within the canopy and that separate biases against feeding in high UV and low green environments exist. Whether this is specific to $M$. persicae or occurs more widely in other species of Hemiptera is not known. M. persicae is present globally and it is also not known how this response would vary through different populations in differing solar conditions. We present a methodology for using prior information of aphid physiological responses to colour to represent spectral measurements in a more intuitive way that could be widely applicable to other species and novel light environments. Whilst this may be of particular interest to applied entomology, particularly for improving pest control under horticultural films, the method could also be of broad interest to those seeking to better understand the relationship between light and behaviours. Future work could consider the breadth of responses through different species and populations, or focus on the genetic mechanisms 
by which behaviour and photoprotection may interact.

\section{Acknowledgements}

The authors are very grateful for the thorough and detailed review of the manuscript by two anonymous reviewers. The authors wish to thank Dr Robert Hardy and Mr Grant Fulton for assistance in construction of the polytunnel structures and experimental laboratory apparatus. Also to Arid Agritec for supply of the polythenes used during the experimental work. Joseph Fennell was supported by a studentship from the Centre for Global Eco-Innovation and Arid Agritec.

\section{Appendix A. Data and Code Repository}

All data and code used to perform analysis and generate plots are available in the git repository https://github.com/joe-fennell/insect_vision_ $2020 /$

\section{Appendix B. Timelapse Video 1}

Timelapse video of a population of Myzus persicae on a leaf with supplementary exposure to a UV-A fluorescent tube https://vimeo.com/382798875

\section{Appendix C. Timelapse Video 2}

Timelapse video of $M$. persicae individuals under two different light treatments of equivalent irradiance https://vimeo.com/382799527

\section{Appendix D. Detailed Aphid Behavioural Measurement Protocol}

Appendix D.1. Experimental setup

Aphids were transferred from glasshouse to laboratory on a leaf from the culture. Mature wingless aphids of approximately similar size and colouration were selected for experiments. A single aphid was transferred by paintbrush directly from the culture plant to an $11 \mathrm{~mm}$ diameter leaf disc placed adaxial side up on a $25 \mathrm{~mm}$ by $25 \mathrm{~mm}$ by $5 \mathrm{~mm}$ open cell foam pad (Figure D.5). For each experimental run, 12 replicate pads were placed in the lid of a standard 96 well assay plate and the lid flooded with distilled water. This prevented 
movement of aphids from one pad to another. The setup procedure was carried out under laboratory fluorescent lighting. The Petri dish or tray was then transferred to the platform underneath the camera and the image capture process started.

\section{Appendix D.2. Imaging equipment}

A Canon 1200D camera fitted with a Canon EF $50 \mathrm{~mm} \mathrm{f} / 2.5$ Compact Macro lens was controlled by a PC using the Astro Photography Tool (http://www.ideiki.com/) software package, which allowed full control of the time-lapse functionality. Images were captured at f/13 with a shutter speed of between $1 / 10$ and $1 / 15$ seconds (depending on treatment). Camera white balance and exposure program was set to Manual to ensure consistent image processing. Cameras captured JPEG images at 30 second intervals for one hour.

\section{Appendix D.3. Software and aphid tracking methods}

The OpenCV $3.0 \mathrm{C}++$ library was used with Python 2.7 bindings to produce general tools for cropping areas of interest, locating the aphid and outputting a calibrated Comma-Separated Value (CSV) file with information relating to aphid position and direction. Python scripts were developed to implement the $\mathrm{C}++$ library and to organise the resulting files. Any required Graphical User Interface (GUI), to allow user-adjustment of detection parameters, were generated using OpenCV. Four key processing steps were identified: image subsetting, spatial calibration, aphid location, and data processing. The software processing steps are described as follows:

\section{Appendix D.4. Image subsetting}

The original image sequences, containing multiple aphid repeats in each, are cropped to produce new image sub-sequences with a single aphid in each (example in Figure D.5.D). This is achieved using a simple interface that allows users to manually identify single aphid areas within the image sequence. All of the files within the original image sequence are then exported as a new subsequence of individual images.

\section{Appendix D.5. Spatial calibration}

Spatial calibration and identification of the boundary of the leaf disc is achieved by generating a GUI displaying an image (Figure D.5.D) from the data folder with a user-defined circle overlaid. The user adjusts the position 


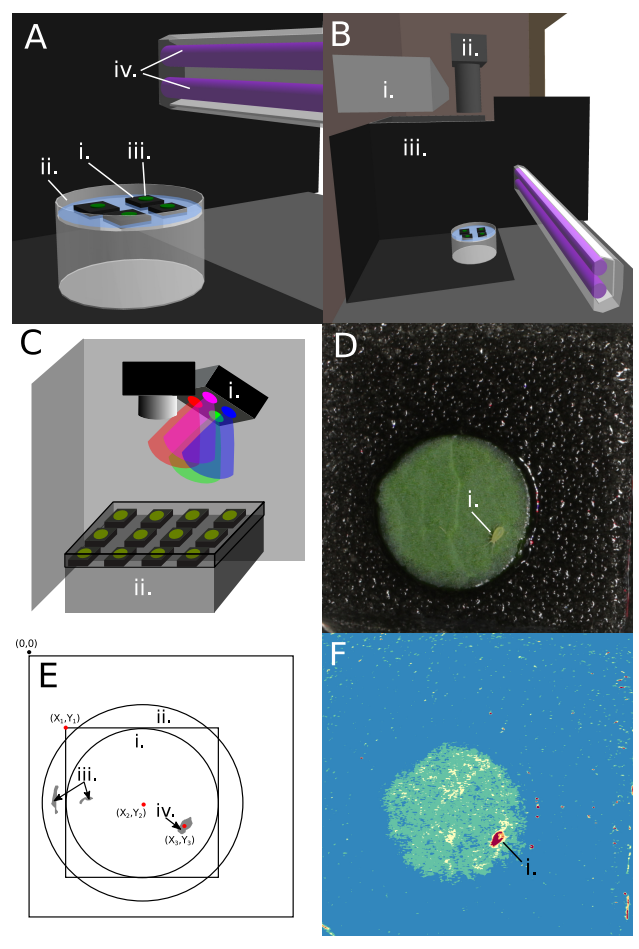

Figure D.5: Image capture and aphid detection stages. In the fluorescent tube supplementation experiments, open-cell foam islands (A.i) were placed in a water filled $90 \mathrm{~mm}$ Petri dish (A.ii) with leaf discs (A.iii) placed on top. Various filtered fluorescent tubes (A.iv) were used to supplement UV with human visible light supplied by a Valoya LED unit (B.i). Images were captured by dSLR cameras (B.ii) mounted directly above the Petri dishes. The two arenas were separated by opaque screens (B.iii). In second set of experiments (LED only), all light was supplied by an LED unit (C.i) and a larger Petri dish was used to allow 12 replicates (C.ii). An example frame is shown pre-analysis as it would be displayed in the GUI (D). (E) Shows the different regions identified by the aphid detection script. Circle (E.i) is the perimeter of the leaf disc expanded by $10 \%$ to generate (E.ii). Non-aphid areas (E.iii) which pass through the colour filter are excluded by size and aspect ratio to correctly identify the centre $\left(X_{3}, Y_{3}\right)$ of the aphid (E.iv) when $X_{1}, Y_{1}=(0,0)$ and $X_{2}, Y_{2}=(5.5,5.5)$. An example frame is shown post-colour filtering (F) to illustrate how colour filtering improves the contrast of the aphid (F.i) against the leaf and background. 
and diameter of the circle to mark the boundary of the $11 \mathrm{~mm}$ leaf disc within a single frame of the image subsequence. The user then views the circle overlaid over the other frames in the subsequence to verify that the boundary is a good fit throughout the image subsequence. Once the diameter and centre coordinates have been confirmed, this information is exported as a JPEG file which is used as a mask image in the Aphid Location processing stage

\section{Appendix D.6. Aphid location}

Each image in an image subsequence is masked using the mask file generated in the previous step. This excludes all areas of the image (excluded area is the area outside the largest circle in Figure D.5.E) from analysis, apart from the leaf disc (Figure D.5.E.iii) and a border zone (Figure D.5.E.ii) to allow detection of aphids on or close to the leaf disc. This masked image is separated into red, green and blue colour channels. To improve aphid contrast with the background, the blue colour channel was subtracted from the red colour channel to produce a single-channel image (figure D.5.F shows falsecolour representation of the single channel image). This is passed through a binary threshold filter with a user-adjustable threshold value to produce a binary (black and white) image.

The binary image is searched for contours (the perimeters of solid white areas in the image) using the OpenCV findContours function. These contours are filtered by minimum size, maximum size and aspect ratio to exclude nonaphid areas (Figure D.5.E.iv) and identify the aphid (Figure D.5.E.v). This is graphically represented with a detection ellipse drawn around the aphid in the GUI. The filter parameters may be adjusted by the user until the aphid is tracked reliably throughout the subsequence.

The centre point of the detection ellipse in each frame is referred to as the aphid's position. During the processing, if no appropriate contour is located or if the position is not within the leaf disc perimeter, the position information is recorded as absent. The pixel positional information is then converted to $X$ and $Y$ values (in $\mathrm{mm}$ ) relative to the top left-hand corner of the square box bounding the leaf disc circle (point $X_{1}, Y_{1}$ in figure D.5.f) and the displacement between current and previous frame is calculated. For two consecutive frames in the subsequence, positional information for both must be present to record a displacement value. If either lack positional information (i.e. the aphid is recorded as off the leaf), displacement is recorded as 
NA in the output file. For each image subsequence, a CSV file is generated containing positional and displacement information for each time interval.

\section{Appendix D.7. Data processing}

Each image subsequence produces a single CSV file in a subfolder. Image subsequences may be (manually) grouped by treatment and sequence during image import. A python script which retrieves all of the individual CSV files and collates them into a single data file and a single summary file was written to facilitate rapid import into statistical software environments.

\section{Appendix D.8. Software calibration}

In order to differentiate normal aphid movement that occurs during feeding (i.e. the wiggle of a feeding aphid) from locomotion, a threshold of 0.014 $\mathrm{mm} \mathrm{s}{ }^{-1}$ was set as a movement threshold to identify time periods when movement was occurring (Figure D.6.A and B). This was set by manually viewing image sequences of aphids in stationary positions after a period of 30 minutes of stationary behaviour. When the aphid was located on the leaf (Figure D.6.C and D) and the velocity was recorded as less than the movement threshold (FigureD.6.A and B), aphids were recorded as in a 'Stationary on Leaf' status (Figure D.6.E and F). The aphid tracking system also allows analysis of the positional information of the aphid over the test period, such as the distance from the leaf disc centre (Figure D.6.G and H).

\section{Appendix E. Light Treatments for lab experiment}

Table of light treatments for lab experiment.

\section{Appendix F. Statistical Analysis Supplementary Information}

Parameter estimates for final field experiment mode (Table F.3, F.4) and laboratory behavioural experiment (Table F.6). Model comparison heuristics using Widely Applicable Information Criterion (WAIC) method as described in Salvatier et al. (2016) (Table F.5).

Model comparison coefficients for laboratory experiment (Table F.5)

Antignus, Y., nov 2000. Manipulation of wavelength-dependent behaviour of insects: an IPM tool to impede insects and restrict epidemics of insectborne viruses. Virus Research 71 (1-2), 213-20. 

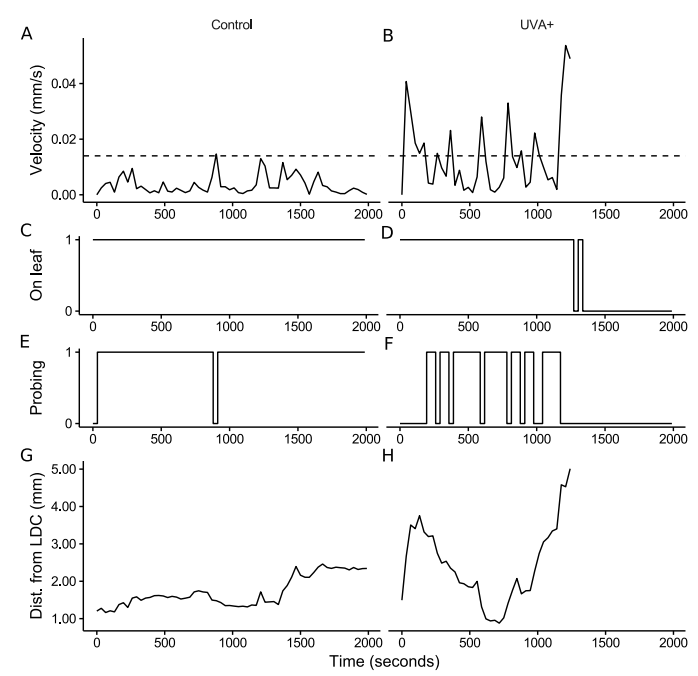

Figure D.6: Aphid tracking raw data. Examples from a control (no ultraviolet-A) and a UVA+ (supplementary ultraviolet-A) LED treatment for single aphids. Traces show two individual aphids under either LED Control (left column) or LED UVA+ (right column) lighting. For each aphid, velocity (A and B), whether or not the aphid was detected on the leaf $(\mathrm{C}$ and $\mathrm{D})$, whether or not this was interpreted as a probing phase ( $\mathrm{E}$ and $\mathrm{F})$ and the aphid distance from leaf Disc Centre $(\mathrm{G}$ and $\mathrm{H})$ is presented against time (seconds). The dashed lines in $(\mathrm{A})$ and $(\mathrm{B})$ show the movement threshold of $0.014 \mathrm{~mm} \mathrm{~s}^{-1}$ 
Table E.2: Light treatments and Myzus persicae visual response coordinates (as described in Section 2.5 used in laboratory experiment. Columns are: (G)reen, (B)lue and UV percentage of max power; aphid photoreceptor total Amplitude; aphid photoreceptor colour coordinates cx and cy (see Section 2.5); and N(umber) of insects measured for a given treatment.

\begin{tabular}{rrrrrrrr}
\hline & G $\%$ & B \% & UV \% & Amplitude & cx & cy & N \\
\hline 1 & 0 & 47 & 0 & 5.519229 & 0.233311 & 0.001130 & 21 \\
2 & 0 & 47 & 49 & 6.349618 & 0.238439 & 0.035882 & 21 \\
3 & 0 & 47 & 100 & 7.186547 & 0.242409 & 0.062782 & 19 \\
4 & 0 & 23 & 0 & 2.759614 & 0.233311 & 0.001130 & 22 \\
5 & 0 & 23 & 49 & 3.590004 & 0.242382 & 0.062597 & 22 \\
6 & 0 & 23 & 100 & 4.426932 & 0.248081 & 0.101215 & 21 \\
7 & 49 & 47 & 0 & 8.194030 & 0.451267 & 0.000996 & 19 \\
8 & 49 & 47 & 49 & 9.024420 & 0.434820 & 0.025460 & 20 \\
9 & 49 & 47 & 100 & 9.861348 & 0.421046 & 0.045949 & 24 \\
10 & 49 & 23 & 0 & 5.434416 & 0.561946 & 0.000928 & 24 \\
11 & 49 & 23 & 49 & 6.264805 & 0.523584 & 0.036178 & 22 \\
12 & 49 & 23 & 100 & 7.101734 & 0.493997 & 0.063364 & 23 \\
13 & 100 & 47 & 0 & 10.889893 & 0.562602 & 0.000928 & 19 \\
14 & 100 & 47 & 49 & 11.720282 & 0.542050 & 0.019770 & 20 \\
15 & 100 & 47 & 100 & 12.557211 & 0.524086 & 0.036239 & 15 \\
16 & 100 & 23 & 0 & 8.130278 & 0.674371 & 0.000859 & 20 \\
17 & 100 & 23 & 49 & 8.960668 & 0.637132 & 0.025510 & 23 \\
18 & 100 & 23 & 100 & 9.797596 & 0.605987 & 0.046127 & 22 \\
19 & 100 & 11 & 49 & 7.580861 & 0.710632 & 0.029948 & 11 \\
20 & 100 & 11 & 100 & 8.417789 & 0.667074 & 0.053503 & 19 \\
21 & 100 & 7 & 15 & 6.552076 & 0.782175 & 0.011401 & 13 \\
\hline
\end{tabular}


Table F.3: Model summary for field experiment final model (Total Population). The columns are parameter estimate ('mean'), standard deviation ('sd') MCMC error, $95 \%$ Credible interval lower estimate ('hpd_2.5'), 95\% Credible interval upper estimate ('hpd_97.5'), Number of effective MC samples ('n_eff') and $\hat{R}$

\begin{tabular}{lrrrrrrr}
\hline & mean & sd & mc_error & hpd_2.5 & hpd_97.5 & n_eff & Rhat \\
\hline Intercept & 5.307 & 0.123 & 0.002 & 5.069 & 5.556 & 5034.459 & 1.000 \\
Cultivar[T.Volta] & -0.132 & 0.127 & 0.001 & -0.377 & 0.111 & 7842.521 & 1.000 \\
LT[T.Standard] & -0.187 & 0.154 & 0.002 & -0.492 & 0.113 & 5679.088 & 1.000 \\
LT[T.UV-opaque] & -0.006 & 0.150 & 0.002 & -0.307 & 0.284 & 5690.884 & 1.000 \\
$\mathrm{mu}$ & 144.619 & 6074.473 & 77.103 & 0.001 & 124.035 & 6095.893 & 1.000 \\
alpha & 5.306 & 1.087 & 0.012 & 3.216 & 7.369 & 7245.257 & 1.000 \\
\hline
\end{tabular}

Table F.4: Model summary for field experiment final model (Exposed Population). The columns are parameter estimate ('mean'), standard deviation ('sd') MCMC error, 95\% Credible interval lower estimate ('hpd_2.5'), 95\% Credible interval upper estimate ('hpd_97.5'), Number of effective MC samples ('n_eff') and $\hat{R}$

\begin{tabular}{lrrrrrrr}
\hline & mean & sd & mc_error & hpd_2.5 & hpd_97.5 & n_eff & Rhat \\
\hline Intercept & 0.858 & 0.260 & 0.004 & 0.341 & 1.365 & 3509.892 & 1.000 \\
Cultivar[T.Volta] & 0.388 & 0.238 & 0.003 & -0.075 & 0.852 & 6086.707 & 1.000 \\
LT[T.Standard] & 0.551 & 0.300 & 0.005 & -0.039 & 1.143 & 4119.907 & 1.000 \\
LT[T.UV-opaque] & 1.190 & 0.283 & 0.004 & 0.627 & 1.740 & 4319.428 & 1.000 \\
mu & 55.277 & 601.187 & 8.038 & 0.001 & 121.881 & 5051.556 & 1.000 \\
alpha & 2.391 & 0.704 & 0.008 & 1.175 & 3.749 & 6546.770 & 1.000 \\
\hline
\end{tabular}


Table F.5: Model comparison, ordered from best to worst model. The columns are Widely Applicable Information Criterion ('WAIC'), estimated number of effective parameters ('pWAIC'), Standard Error of WAIC estimate ('SE') and the model formulae

\begin{tabular}{lllll}
\hline & WAIC & pWAIC & SE & model formulae \\
\hline 8 & 560.71 & 3.03 & 10.55 & $y \sim c x+c y$ \\
5 & 562.71 & 4.11 & 10.54 & $y \sim A+c x+c y$ \\
11 & 562.94 & 2.11 & 9.7 & $y \sim c y$ \\
2 & 563.94 & 5.05 & 10.63 & $y \sim(A * c y)+(c x)$ \\
1 & 564.34 & 5.05 & 10.7 & $y \sim(A * c x)+(c y)$ \\
7 & 564.38 & 3.12 & 9.86 & $y \sim A+c y$ \\
0 & 565.64 & 6.1 & 10.79 & $y \sim(A * c y)+(A * c x)$ \\
4 & 565.71 & 4.08 & 9.94 & $y \sim(A * c y)$ \\
10 & 575.79 & 1.98 & 6.44 & $y \sim c x$ \\
6 & 577.56 & 3.04 & 6.6 & $y \sim A+c x$ \\
3 & 579.66 & 4.17 & 6.66 & $y \sim(A * c x)$ \\
9 & 584.75 & 2.04 & 2.44 & $y \sim A$ \\
\hline
\end{tabular}

Table F.6: Model summary for laboratory experiment final model (Avoidance Response). The columns are parameter estimate ('mean'), standard deviation ('sd') MCMC error, 95\% Credible interval lower estimate (' $h p d_{-} 2.5$ '), 95\% Credible interval upper estimate ('hpd_97.5'), Number of effective MC samples ('n_eff') and $\hat{R}$

\begin{tabular}{lrrrrrrr}
\hline & mean & sd & mc_error & hpd_2.5 & hpd_97.5 & n_eff & Rhat \\
\hline Intercept & 0.088 & 0.355 & 0.006 & -0.636 & 0.742 & 2902.333 & 1.000 \\
cx & -1.296 & 0.619 & 0.010 & -2.489 & -0.072 & 3065.916 & 1.000 \\
cy & 16.362 & 3.999 & 0.052 & 8.414 & 24.100 & 4435.181 & 1.000 \\
\hline
\end{tabular}


ASTM International, 2012. Standard Tables for Reference Solar Spectral Irradiances: Direct Normal and Hemispherical on 37 Tilted Surface. URL https : //www . astm . org/Standards/G173.htm

Ballaré, C. L., jan 2014. Light Regulation of Plant Defense. Annual review of plant biology.

URL http://www .ncbi.nlm.nih.gov/pubmed/24471835

Barta, A., Horváth, G., feb 2004. Why is it advantageous for animals to detect celestial polarization in the ultraviolet? Skylight polarization under clouds and canopies is strongest in the UV. Journal of Theoretical Biology 226 (4), 429-437.

URL http://www.ncbi.nlm.nih.gov/pubmed/14759649

Borst, A., jan 2009. Drosophila's view on insect vision. Current biology : CB 19 (1), R36-47.

URL http://www.ncbi.nlm.nih.gov/pubmed/19138592

Briscoe, A. D., Chittka, L., 2001. The evolution of color vision in insects. Annual Review of Entomology 46, 471-510.

Burdick, S. C., Prischmann-Voldseth, D. A., Harmon, J. P., 2015. Density and distribution of soybean aphid, Aphis glycines Matsumura (Hemiptera: Aphididae) in response to UV radiation. Population Ecology 57 (3), 457466.

Chittka, L., Beier, W., Hertep, H., Steinmann, E., Menzel, R., 1992. Opponent colour coding is a universal strategy to evaluate the photoreceptor inputs in Hymenoptera. Journal of Comparative Physiology A: Sensory, Neural, and Behavioral Physiology 170, 545-563.

Chyzik, R., Dobrinin, S., Antignus, Y., 2003. Effect of a UV-deficient environment on the biology and flight activity of Myzus persicae and its hymenopterous parasite aphidius matricariae. Phytoparasitica 31 (5), 467477.

Costa, H. S., Robb, K. L., Heather S. Costa, K. L. R., 1999. Effects of ultraviolet-absorbing greenhouse plastic films on flight behavior of Bemisia argentifolii (Homoptera : Aleyrodidae) and Frankliniella occidentalis (Thysanoptera : Thripidae). Journal of Economic Entomology 92 (3), 557562 . 
Döring, T. F., Chittka, L., Doring, T. F., mar 2007. Visual ecology of aphidsa critical review on the role of colours in host finding. Arthropod-Plant Interactions 1 (1), 3-16. URL http://link.springer.com/10.1007/s11829-006-9000-1

Doring, T. F., Kirchner, S. M., 2007. Preliminary characterisation of the spectral sensitivity in the cabbage aphid with electroretinogram recordings (Hemiptera : Aphididae). Entomologia Generalis 30 (3), 233-234.

Doring, T. F., Kirchner, S. M., Kuhne, S., Saucke, H., Döring, T. F., Kühne, S., 2004. Response of alate aphids to green targets on coloured backgrounds. Entomologia Experimentalis Et Applicata 113 (1), 53-61.

Egelhaaf, M., Kern, R., dec 2002. Vision in flying insects. Current Opinion in Neurobiology 12 (6), 699-706.

URL http://linkinghub.elsevier.com/retrieve/pii/ S0959438802003902

Fennell, J., 2016. Behavioural and Physiological Responses of Myzus persicae to Ultraviolet Light for the Development of New Pest Control Technologies. Ph.D. thesis, Lancaster University, Lancaster, United Kingdom.

Fennell, J. T., Fountain, M. T., Paul, N. D., 2019. Direct effects of protective cladding material on insect pests in crops. Crop Protection 121, 147 156.

URL http://www.sciencedirect.com/science/article/pii/ S0261219419301073

Fukaya, M., Uesugi, R., Ohashi, H., Sakai, Y., Sudo, M., Kasai, A., Kishimoto, H., Osakabe, M., 2013. Tolerance to Solar Ultraviolet-B Radiation in the Citrus Red Mite, An Upper Surface User of Host Plant Leaves. Photochemistry and Photobiology 89 (2), 424-431.

Gitelson, A. A., Chivkunova, O. B., Merzlyak, M. N., oct 2009. Nondestructive estimation of anthocyanins and chlorophylls in anthocyanic leaves. American Journal of Botany 96 (10), 1861-1868. URL http://www . amjbot .org/cgi/doi/10.3732/ajb.0800395

Giurfa, M., Menzel, R., aug 1997. Insect visual perception: complex abilities of simple nervous systems. Current Opinion in Neurobiology 7 (4), 
505-513.

Golawska, S., Lukasik, I., Goławska, S., dec 2012. Antifeedant activity of luteolin and genistein against the pea aphid, Acyrthosiphon pisum. Journal of Pest Science 85 (4), 443-450.

URL http://www.pubmedcentral.nih.gov/articlerender.fcgi? artid=3505511\{\&\}tool=pmcentrez $\{\&\}$ rendertype=abstract

Hardie, J., Holyoak, M., Taylor, N. J., Griffiths, D. C., 1992. The combination of electronic monitoring and video-assisted observations of plant penetration by aphids and behavioural effects of polygodial. Entomologia Experimentalis et Applicata 62 (3), 233-239.

Johnson, E. T., Dowd, P. F., aug 2004. Differentially enhanced insect resistance, at a cost, in Arabidopsis thaliana constitutively expressing a transcription factor of defensive metabolites. Journal of agricultural and food chemistry 52 (16), 5135-8.

URL http://www.ncbi.nlm.nih.gov/pubmed/15291486

Kigathi, R., Poehling, H.-M. M., dec 2012. UV-absorbing films and nets affect the dispersal of western flower thrips, Frankliniella occidentalis (Thysanoptera: Thripidae). Journal of Applied Entomology 136 (10), 761771.

URL http://doi.wiley.com/10.1111/j.1439-0418.2012.01707.x

Kirchner, S. M., Döring, T. F., Saucke, H., Doring, T. F., nov 2005. Evidence for trichromacy in the green peach aphid, Myzus persicae (Sulz.) (Hemiptera: Aphididae). Journal of insect physiology 51 (11), 1255-60. URL http://www.ncbi.nlm.nih.gov/pubmed/16162354

Langley, S. a., Tilmon, K. J., Cardinale, B. J., Ives, A. R., nov 2006. Learning by the parasitoid wasp, Aphidius ervi (Hymenoptera: Braconidae), alters individual fixed preferences for pea aphid color morphs. Oecologia 150 (1), 172-9.

URL http://www.ncbi.nlm.nih.gov/pubmed/16858585

Legarrea, S., Betancourt, M., Plaza, M., Fraile, a., Garcia-Arenal, F., Fereres, A., García-Arenal, F., Fereres, A., apr 2012a. Dynamics of nonpersistent 
aphid-borne viruses in lettuce crops covered with UV-absorbing nets. Virus Research 165 (1), 1-8. URL http://www.ncbi.nlm.nih.gov/pubmed/22226944

Legarrea, S., Weintraub, P. G., Plaza, M., Viñuela, E., Fereres, A., Vinuela, E., Fereres, A., Viñuela, E., Fereres, A., Vinuela, E., dec 2012b. Dispersal of aphids, whiteflies and their natural enemies under photoselective nets. BioControl 57 (4), 523-532.

Möller, R., feb 2002. Insects could exploit UV-green contrast for Landmark navigation. Journal of theoretical biology 214 (4), 619-31. URL http://www .ncbi .nlm.nih.gov/pubmed/11851371

Ohtsuka, K., Osakabe, M. M., jun 2009. Deleterious Effects of UV-B Radiation on Herbivorous Spider Mites: They Can Avoid It by Remaining on Lower Leaf Surfaces. Environmental Entomology 38 (3), 920-929.

URL http: //apps.webofknowledge.com/

CitedFullRecord. do?product $=W O S\{\&\}$ colName $=$

WOS $\{\&\}$ SID $=$ T1J9sZ5UA5wz32153xD $\{\&\}$ search $\left\{\_\right\}$mode $=$

CitedFullRecord $\{\&\}$ isickref=WOS : 000266655800046

Onzo, A., Sabelis, M. W., Hanna, R., apr 2010. Effects of ultraviolet radiation on predatory mites and the role of refuges in plant structures. Environmental entomology 39 (2), 695-701. URL http://www .ncbi.nlm.nih.gov/pubmed/20388304

Osorio, D., Vorobyev, M., sep 2008. A review of the evolution of animal colour vision and visual communication signals. Vision research 48 (20), 2042-51. URL http://www.ncbi.nlm.nih.gov/pubmed/18627773

Paul, N. D., Jacobson, R. J., Taylor, A., Wargent, J. J., Moore, J. P., 2005. The use of wavelength-selective plastic cladding materials in horticulture: understanding of crop and fungal responses through the assessment of biological spectral weighting functions. Photochemistry and photobiology 81 (5), 1052-60.

URL http://www .ncbi.nlm.nih.gov/pubmed/15819600

Paulsen, C., Cottrell, T., Ruberson, J., feb 2013. Distribution of the black pecan aphid, Melanocallis caryaefoliae, on the upper and lower surface of 
pecan foliage. Entomologia Experimentalis et Applicata 146 (2), 252-260. URL http://doi.wiley.com/10.1111/eea.12018

Pfeiffer, K., Homberg, U., jun 2007. Coding of Azimuthal Directions via Time-Compensated Combination of Celestial Compass Cues. Current Biology 17 (11), 960-965.

URL http://www .ncbi.nlm.nih.gov/pubmed/17524646

Rangasamy, M., McAuslane, H. J., Backus, E. A., Cherry, R. H., feb 2015. Differential Probing Behavior of Blissus insularis (Hemiptera: Blissidae) on Resistant and Susceptible St. Augustinegrasses. Journal of Economic Entomology 108 (2), 780-788. URL http://jee.oxfordjournals.org/content/108/2/780.abstract

Raviv, M., Antignus, Y., Yishay, R., 2004. Invited Review UV Radiation Effects on Pathogens and Insect Pests of Greenhouse-Grown Crops. Photochemistry and Photobiology 79 (3), 219-226.

Sakai, Y., Sudo, M., Osakabe, M., jan 2012. Seasonal changes in the deleterious effects of solar ultraviolet-B radiation on eggs of the twospotted spider mite, Tetranychus urticae (Acari: Tetranychidae). Applied Entomology and Zoology 47 (1), 67-73.

URL http://link . springer .com/10.1007/s13355-011-0090-6

Salvatier, J., Wiecki, T. V., Fonnesbeck, C., apr 2016. Probabilistic programming in python using PyMC3. PeerJ Computer Science 2, e55.

URL https://doi.org/10.7717/peerj-cs.55

Simmons, A. M., apr 1999. Nymphal survival and movement of crawlers of Bemisia argentifolii (Homoptera: Aleyrodidae) on leaf surfaces of selected vegetables. Environmental entomology 28 (2), 212-6. URL http://www.ncbi.nlm.nih.gov/pubmed/11543187

Tariq, K., Noor, M., Saeed, S., Zhang, H., 2015. The Effect of Ultraviolet-A Radiation Exposure on the Reproductive Ability, Longevity, and Development of the $<\mathrm{i}>$ Dialeurodes citri $</ \mathrm{i}>$ (Homoptera: Aleyrodidae) F1 Generation. Environmental Entomology 44 (6), 1614-1618.

URL http://ee.oxfordjournals.org/lookup/doi/10.1093/ee/ nvv133 
781

782

783

784

785

Ver Hoef, J. M., Boveng, P. L., 2007. Quasi-poisson vs. negative binomial regression: How should we model overdispersed count data? Ecology 88 (11), 2766-2772.

URL https://esajournals.onlinelibrary.wiley.com/doi/abs/10. 1890/07-0043.1

Zu-Qing, H., Hui-Yan, Z., Thomas, T., 2013. Probing behaviors of Sitobion avenae (Hemiptera: Aphididae) on enhanced UV-B irradiated plants. Archives of Biological Sciences 65 (1), 247-254.

URL

http://www.doiserbia.nb.rs/Article.aspx?ID= 0354-46641301247H 